\title{
Política y cambio en educación: el caso de las competencias básicas en España $^{1}$
}

\author{
Héctor Monarca ${ }^{a}$ \\ Cecilia Simón ${ }^{b}$ \\ Soledad Rappoport ${ }^{\mathrm{c}}$ \\ Gerardo Echeita ${ }^{d}$
}

\section{Resumen}

Este artículo presenta los resultados de una investigación cuyo objetivo ha sido analizar la forma en que las políticas públicas producen o no cambios en los ámbitos de la realidad social a la que se dirigen. Se aborda específicamente el cambio normativo que ha regulado la implementación del enfoque pedagógico de competencias en España. Se asume una concepción procesual de las políticas, las cuales se estudian aquí a partir de la mirada de diversos actores del nivel micro. Se empleó una metodología cualitativa, realizando un estudio de casos múltiples en centros de educación primaria y secundaria de tres comunidades autónomas diferentes. Los resultados muestran una distancia considerable entre las pretensiones normativas y lo que realmente ha terminado sucediendo dentro de las aulas de los centros educativos. Las conclusiones sugieren una necesaria reflexión sobre la forma en que se conciben y desarrollan las políticas públicas.

Palabras clave: Política educativa. Cambio educativo. Competencias básicas. Reformas educativas. Estudio de casos.

\footnotetext{
a Universidad Autónoma de Madrid, Departamento de Didáctica y Teoría de la Educación. Madrid, España.

b Universidad Autónoma de Madrid, Departamento Interfacultativo de Psicología Evolutiva y de la Educación. Madrid, España.

c Universidad Autónoma de Madrid, Departamento de Didáctica y Teoría de la Educación. Madrid, España.

d Universidad Autónoma de Madrid, Departamento Interfacultativo de Psicología Evolutiva y de la Educación. Madrid, España.
}

Recebido em: 05 out. 2015

Aceito em: 22 jan. 16

\footnotetext{
El presente artículo deriva de los resultados del «Estudio de las dimensiones curriculares, organizativas, metodológicas y evaluativas para el desarrollo de la competencia social y ciudadana en Educación Primaria y Educación Secundaria Obligatoria», financiado por el Ministerio de Educación, Cultura y Deporte de España, de acuerdo con la Resolución de 20 de octubre de 2011, de la Secretaría de Estado de Educación y Formación Profesional, por la que se conceden ayudas para los proyectos que profundicen en la consolidación de las competencias básicas como elemento esencial del currículo, al amparo de la Orden EDU/1427/2011, de 20 de mayo, por la que se crearon y se convocaron dichas ayudas en régimen de concurrencia competitiva.
} 


\section{Introducción}

En el año 2006, el Ministerio de Educación de España promovió un nuevo cambio educativo regulado normativamente en la Ley Orgánica 2/2006, de 3 de mayo, de Educación (ESPAÑA, 2006). Asumiendo las recomendaciones de la Comisión y del Parlamento Europeo, uno de los cambios más significativos que ha propiciado fue la incorporación de las competencias básicas como nuevo componente del currículo y nuevo enfoque pedagógico. Posteriormente, las Comunidades Autónomas desarrollaron su propia normativa, asumiendo el nivel de concreción curricular que tienen asignado. Finalmente, han sido los centros educativos quienes, de acuerdo con los niveles de concreción estipulados en la normativa, han tenido que transformar estas prescripciones en prácticas educativas.

De acuerdo con esta lógica normativa de concreción curricular (GIMENO, 1988), y de la investigación relacionada con el cambio educativo (FULLAN, 2002; GIMENO, 2006; HARGREAVES, 1996; MOYA; LUENGO, 2011; PERRENOUD, 2012; SARASON, 2003; VIÑAO, 2002; 2006), para que la incorporación de este nuevo componente, y del nuevo enfoque pedagógico asociado al mismo, se transforme en una realidad en la vida cotidiana de los centros, necesita de acciones a varios niveles de forma simultánea relacionadas con: a) la normativa y otros documentos de concreción de la misma; b) información, formación, asesoramiento y acompañamiento por parte de la Administración educativa; c) la inspección educativa, dentro de las anteriores; y d) acciones específicas diseñadas y promovidas por los centros: elaboración de documentos, información, formación, debates, etc. Desde una lógica compleja, sistémica, procesual y participativa de las políticas públicas (BUENFIL, 2006; NICASTRO, 2010; RAMÍREZ, 2010; SHORE, 2010; VITAR, 2006), cada una de estas actuaciones son esenciales para pasar de una pretensión normativa al cambio de las realidades a las que estas se dirigen.

Es conveniente mencionar que este proceso de cambio se ubica en un contexto especialmente inestable con respecto a los proyectos educativos, plasmados en propuestas normativas diferenciadas, que se han ido generando en los últimos años. En este sentido, la literatura señala que la gran cantidad de cambios normativos que han tenido lugar en el contexto español, y a los que se ha tenido que enfrentar el profesorado, han influido negativamente en la misma recepción de estas propuestas, viviéndose con mucha frecuencia como una mera exigencia burocrática, sin trascendencia para las prácticas escolares (ÁLVAREZ, 2008; BOLIVAR, 2008; DÍEZ HERNANDO, 2008; PUELLES, 2006).

En cualquier caso, no se puede perder de vista que los centros configuran verdaderas culturas (HARGREAVES, 1996; PÉREZ GÓMEZ, 1998; VIÑAO, 2002), que no 
pueden ser modificadas por el solo hecho de prescribir. La literatura enmarcada dentro de esta tradición interpretativa y crítica, ubica los cambios en procesos de negociación, resignificación y construcción compartida y colaborativa de las prácticas sociales y educativas (FULLAN, 2002; MONARCA, 2013; MURILLO Y KRICHESKY, 2012; VIÑAO, 2006).

En esta línea, este artículo presenta los resultados de una investigación cuyo objetivo ha sido, precisamente, indagar sobre los procesos que se han generado en los centros educativos a partir de la prescripción normativa relacionada con la incorporación de las competencias básicas, y conocer los cambios que dichos procesos promovieron.

\section{El currículo como marco de los cambios en educación}

A pesar de los años que se lleva debatiendo en torno al currículo como «organizador de las enseñanzas», siguen existiendo grandes diferencias en las concepciones que giran en torno al mismo. Estas se relacionan con las teorías y los paradigmas desde los cuales se introducen en esta temática, las cuales, como en cualquier otro caso, hacen de prisma para interpretarlo y pronunciarse en torno a él, para definirlo y construirlo (SCHÜTZ, 2003). Para su comprensión, se hace necesaria una aproximación desde un pensamiento complejo (MORIN, 2003), que evite miradas simplistas y reduccionistas, y permita ubicarlo en relación con diversas prácticas que lo atraviesan, condicionan y definen (GIMENO, 1988). Por eso, ante todo, el currículo debe ser visto como una construcción social y cultural en la que intervienen, además de las prácticas mencionadas, múltiples actores; razón por la cual, lo más adecuado es apreciarlo desde una concepción procesual en la que el currículo va cobrando existencia.

Hace algún tiempo que el currículo es para los gobiernos un dispositivo privilegiado a la hora de encarar las reformas educativas, empleándolo como medio para organizar las intenciones educativas asociadas a un contexto de demandas diversas. En este sentido, son muchos los que hablan de un nuevo contexto social (CASTELLS, 1997; DUBET, 2006) producto de grandes cambios tecnológicos, nuevas formas y usos de la comunicación y la información, cambios en las relaciones entre la sociedad y el conocimiento, y cambios en las instituciones; nuevas demandas que conviven con otras ya existentes. Estos cambios plantean, en muchos casos, nuevos desafíos y presiones sobre lo que se espera de la educación y de la enseñanza, donde coexisten tendencias reproductoras con otras productoras, creativas e innovadoras (GIMENO, 2005). En este marco, es en el currículo como campo de «disputas e intereses», como objetivación de «mandatos» (DALE, 1989), donde se ubica la nueva dimensión que se ha dado a los currículos, a la enseñanza y al aprendizaje, con la inclusión de las denominadas competencias básicas. 
El currículo prescrito refleja estas presiones sociales, culturales, económicas e históricas (GOODSON, 2010), las cuales se trasladan a los centros y a los profesores. Se constituye así un complejo panorama con intereses y visiones -que no siempre son coincidentes- sobre el mundo, el conocimiento, la educación y sus funciones. Estas presiones, tensiones y demandas se aprecian en los distintos elementos de la cultura que se ha decidido, en complejos procesos históricos y de toma de decisiones, que formen parte, en el caso concreto de España, de la enseñanza básica y común: conocimientos disciplinares o de diversos campos, valores, procedimientos y otros conocimientos que se han considerado relevantes (GOODSON, 1998), incorporándose recientemente un nuevo elemento, las competencias.

Esto también se ve reflejado en el complejo proceso de concreción curricular que realizan los centros educativos en el profesorado: su pensamiento y su discurso, en las acciones educativas que despliegan; en las demandas y expectativas de los padres; en los alumnos como partícipes del proceso de enseñanza y de aprendizaje; así como en todos los elementos que atraviesan y condicionan los contextos de enseñanza.

De esta manera, hay que ubicar a las competencias básicas en este complejo proceso, que va desde las prescripciones normativas, hasta la forma real que asumen en los contextos escolares. Por otra parte, de alguna manera, este nuevo componente del currículo, y el nuevo enfoque asociado al mismo, interpela al currículo anterior, al currículo centrado en las disciplinas. Los rasgos que las definen demandan nuevos formatos, una visión que trascienda la lógica disciplinar. Desde el punto de vista pedagógico, el enfoque de competencias exige cambios de miradas y de formas de hacer; la educación actual, tal como ha sido institucionalizada, el peso de su historia y la coexistencia de demandas y tradiciones diversas, incluso, contradictorias, es interpelada por este nuevo enfoque pedagógico.

\section{Políticas orientadas a reformas, cambios o mejoras educativas}

Dentro del estudio de las políticas públicas se ha prestado especial atención a aquellas que han promovido reformas, intentos de cambio o mejora educativa, tanto en el contexto internacional como en el español (ADELMAN; WALKINGEAGLE, 2003; ÁLVAREZ, 2008; DIEZ, 2008; DONOSO; DONOSO, 2009; FULLAN, 2002; GIMENO, 2006; HARGREAVES, 1996; PERRENOUD, 2012; PUELLES, 2006; SARASON, 2003; VIÑAO, 2002, 2006). Existe una importante coincidencia en la distancia que suele existir entre las propuestas de cambio y los cambios que efectivamente se producen. Posiblemente, sobre todo desde el 
enfoque procesual y etnográfico asumido, este hecho deba ser entendido como normal, ya que las evidencias muestran que las reformas, los cambios y las mejoras nunca son producto de la aplicación lineal y mecánica de un diseño previo.

En este sentido, un aspecto que menciona la literatura es la identificación de dos dimensiones del cambio: una objetiva y otra subjetiva (FULLAN, 2002). Desde el punto de vista individual, este autor considera relevante que las propuestas de cambio ofrezcan a los docentes la oportunidad de reflexionar sobre sus hábitos y creencias con el fin de modificarlos, proceso que denomina «reculturización». Fullan (2002) considera que estos procesos son más eficaces que las frecuentes medidas de cambio que apuntan, en lugar de a la cultura de los centros, a las estructuras de los mismos; proceso que, en este caso, denomina «reestructuración». Por otra parte, desde el punto de vista objetivo, el autor sostiene que estos procesos de cambio tienen un carácter multidimensional, y menciona tres dimensiones clave que se debe tener en cuenta a la hora de desarrollar nuevas políticas: a) recursos didácticos, b) enfoques metodológicos, y c) creencias.

Otro aspecto que ha destacado la investigación sobre las reformas educativas, concretamente sobre la normativa que se prescribe, es que muchas veces se formula en términos excesivamente simplificadores (PERRENOUD, 2012). Se alude para ello que estas regulaciones o prescripciones deben contemplar un público amplio y diversos escenarios, lo cual deriva en formulaciones fundamentalmente de carácter abstracto, genéricas, en ocasiones con escasa o ninguna concreción específica que oriente en los cambios que intentan promover. Siendo así, estas simplificaciones no se constituyen en una guía, orientación o en un apoyo para las prácticas educativas; incluso, en ocasiones, no solo no constituyen una guía, sino que pueden ser fuente de confusión para el objetivo que estas normas proponen, sobre todo, cuando no va unido a otros procesos (PERRENOUD, 2012). Por otra parte, estas simplificaciones suelen ir acompañadas de un pobre desarrollo conceptual, algo que se ha cuestionado especialmente con respecto a la introducción del enfoque de competencias, tanto en España como en otros países.

La investigación sobre el cambio educativo destaca también la importancia del trabajo colaborativo y los procesos participativos en general. Especial importancia se ha dado al trabajo colaborativo entre profesores (MURILLO; KRICHESKY, 2012; PARRILA, 2004), a la participación de las familias (MONARCA; SIMÓN, 2013), a la participación de los estudiantes y al trabajo cooperativo entre ellos (PUJALÀS, 2009).

Desde este punto de vista, hay coincidencia en la necesidad de un acercamiento de posturas, un intercambio acerca de las concepciones y sentidos que los sujetos 
tienen sobre la educación y sobre los cambios propuestos, para poder configurar así un escenario de significados compartidos donde los cambios y las mejoras sean posibles. También hay coincidencia en que si estos procesos no tienen cabida, en la mayoría de los casos no se produce ningún cambio significativo.

Finalmente, aparece otro elemento que ha sido puesto de manifiesto en numerosas investigaciones: el lugar del «tiempo» en las reformas o procesos de cambio (ADELMAN; WALKING-EAGLE, 2003; PERRENOUD, 2012). El último de estos autores considera que las reformas suelen caracterizarse por exigir una velocidad que atenta contra los procesos de discusión, reflexión, lectura, que son imprescindibles para generar las condiciones adecuadas a partir de las cuales desarrollar reformas en general, o cualquier otro proceso de cambio. Por su parte, las investigadoras Adelman y Walking-Eagle (2003), a partir de su investigación sobre las relaciones entre las innovaciones y el tiempo, han identificado tres etapas relacionadas con el cambio:

- Una primera etapa en la que los docentes deben tener tiempo para conocer y practicar la nueva propuesta. Esta etapa se caracteriza por ser considerada fundamentalmente como un «escenario de prueba», alejado de cualquier mirada sancionadora, en la cual, de acuerdo con estas autoras, «vale equivocarse».

- Una etapa en la que los docentes necesitan tiempo para la implementación, ahora sí para institucionalizar el cambio, es decir, para hacerlo parte de la vida del centro y de las aulas. En este caso, el proceso entra en una fase en la que empieza a «formalizarse», es decir, a asumir un rasgo institucional.

- Por último, identifican una etapa en la que el profesorado precisa tiempo para la reflexión y la evaluación para continuar con la nueva propuesta. En esta última etapa resulta esencial valorar lo realizado, consolidar lo que ha funcionado y seguir cambiando en aquellos aspectos que la evaluación indique.

Según las evidencias de Adelman y Walking-Eagle (2003), estas etapas son claves para conseguir las mejoras propuestas. Al igual que otros autores, entienden que cualquier propuesta de cambio necesita tiempos que, según se deduce de su propia investigación, deben ser contemplados en los mismos procesos de cambio y planificados dentro de las políticas que los promueven. Por otra parte, dentro de este tiempo de preparación previo a la implementación, y en coincidencia con otros investigadores, le dan importancia a la comprensión de la propuesta por parte de los actores 
implicados, de los objetivos que contempla, de las mejoras que va a aportar (FULLAN, 2002; HARGREAVES, 1996; PERRENOUD, 2012).

En cualquier caso, un aspecto que destacan en sus investigaciones es que existen distintos ritmos de cambio, aspecto que es necesario admitir y tener en cuenta en los procesos de reforma y cambio educativo. Es lógico pensar que las instituciones escolares tienen historias particulares, se ubican en contextos singulares, y atienden a poblaciones específicas, lo cual va configurando escenarios diversos para el desarrollo de cambios e innovaciones educativas. Desconocer este hecho y pretender que todas se embarquen en determinados procesos de cambio y mejora a la misma velocidad y con la misma intensidad, puede ser un obstáculo en sí mismo para que estos tengan lugar.

\section{Método}

Para conseguir el objetivo explicado en la introducción, la investigación que aquí se presenta se desarrolló mediante una metodología cualitativa, realizando un estudio de casos múltiple o colectivo, instrumental, de tipo descriptivointerpretativo (STAKE, 2006).

\subsection{Casos}

Para el estudio se escogieron seis centros educativos, tres de educación primaria y tres de educación secundaria obligatoria, ubicados en tres Comunidades Autónomas diferentes. Los centros fueron elegidos de acuerdo con los siguientes criterios: a) centros similares en tamaño, contexto y características de alumnado; b) centros ubicados en comunidades autónomas diferentes; c) centros que destacaran a nivel del entorno por algún rasgo valorado positivamente; y d) centros que permitiesen el acceso prolongado y sistemático del investigador.

\subsection{Modelo de aproximación al objeto de estudio}

En coherencia con el marco teórico, se ha contemplado un modelo de análisis procesual formado por cinco niveles, los cuales contribuyen a focalizar la atención en aspectos específicos del objeto de estudio:

1. Nivel I: el currículo prescrito, constituido por la normativa que fija las competencias como nuevo componente curricular, tanto a nivel estatal como de las Comunidades Autónomas. 
2. Nivel II: las acciones desarrolladas por la Administración para incorporar las competencias clave. Contempla todas aquellas acciones y prácticas que la Administración ha puesto en marcha con el objetivo de favorecer la implementación del enfoque de competencias.

3. Nivel III: las acciones desarrolladas por los centros para incorporar las competencias clave. Se refiere a las acciones centradas en la elaboración de los documentos del centro, a los documentos en sí mismos, y a cualquier otro tipo de acción o práctica relacionada con la implementación de las competencias.

4. Nivel IV: el currículo en acción. Comprende las acciones, programas y proyectos, desarrollados por el profesorado y el centro.

5. Nivel V: el currículo evaluado. Se refiere a las prácticas y acciones de evaluación de las competencias.

\subsection{Técnicas y procedimientos}

De acuerdo con la opción metodológica realizada, se han empleado las siguientes técnicas de investigación:

a) Entrevistas, un total de 76 . De ellas, 15 han sido holísticas, es decir, destinadas a suministrar contexto (VELASCO y DÍAZ DE RADA, 1999), y las 61 entrevistas restantes han sido focalizadas en el objeto de estudio.

b) Observaciones descriptivas/abiertas y focalizadas/selectivas, algunas de las cuales han sido participativas.

c) Análisis de documentos. Se han contemplado dos tipos principales de documentos:

1. Prescriptivos: I) Nivel estatal: a) Ley Orgánica de Educación (LOE); b) Real Decreto 1513/2006, de 7 de diciembre, por el que se establecen las enseñanzas mínimas de la Educación primaria; y c) Real Decreto 1631/2006, de 29 de diciembre, por el que se establecen las enseñanzas mínimas correspondientes a la Educación Secundaria Obligatoria. II) Nivel Autonómico: la normativa específica de cada una de las Comunidades Autónomas escogidas.

2. Elaborados por el profesorado: a) Proyecto educativo de centro (PEC); b) Programación general anual (PGA); c) Plan de atención a la diversidad 
(PAD); d) Plan de acción tutorial (PAT); e) Programaciones didácticas; y f) otros planes o programas, según los casos: planes de convivencia, plan de diversificación curricular, memorias anuales, etc.

\subsection{Trabajo de campo}

El trabajo de campo se ha desarrollado a lo largo de un curso escolar completo. El mismo se ha dividido en dos fases principales, y una tercera, importante desde el punto de vista del compromiso como investigadores, pero no esencial en el estudio en sí mismo. La idea fue iniciar un proceso de aproximación progresiva a nuestro objeto de investigación a través de las dos primeras fases mencionadas.

Fase 1: Aproximación holística. En esta primera fase el objetivo fue acceder a la "cultura" del centro desde un punto de vista más general y holístico, no desde el punto de vista específico del objeto de la investigación. Esta primera fase de estancia en los centros ha tenido tres objetivos: a) construir esta visión holística para identificar posibles «ámbitos»o «áreas» de indagación más específicas; b) identificar posibles informantes, personas relevantes desde el punto de vista del discurso específico relacionado con el objeto de estudio; y c) construir una relación de confianza y cercanía, imprescindible para el acceso a la cultura. En esta fase se han negociado algunos aspectos del acceso al campo (VELASCO y DÍAZ DE RADA, 1999). Entre otros acuerdos, se decidió que, conforme a las circunstancias y el punto de vista del investigador, se podrían asumir roles más o menos participantes dentro de la vida cotidiana del centro.

Fase 2: Profundización y focalización. Esta fase se ha centrado en los objetivos específicos de la investigación. El «discurso emic» sobre el que se ha indagado, profundizado, es el que se relaciona con las competencias básicas, con la forma en que las prescripciones normativas relacionadas con las mismas habían sido apropiadas, resignificadas y transformadas en acciones. En esta fase, al principio se ha dedicado más tiempo a observar, y posteriormente a entrevistar.

Fase 3: Devolución y aportes. Esta última fase ha tenido como objetivo realizar un aporte al centro a partir del trabajo de investigación realizado. Además de un informe específico, el aporte ha sido diverso: a) se ha concretado alguna demanda vinculada con las competencias, b) el centro ha solicitado participación en alguna reunión en la que se abordó esta temática, c) se ha pedido colaboración en alguna actividad relacionada con alguna competencia específica, etc.

Explicadas estas tres fases, es preciso aclarar que las mismas no siempre se pueden ver como claramente diferenciables. Los límites entre las mismas son 
difusos y han funcionado fundamentalmente como organizadoras de intenciones y acciones relacionadas con el trabajo de campo. Sin embargo, a lo largo del desarrollo de este trabajo, aunque a grandes rasgos se ha mantenido la estructura de fases mencionada, se han dado circunstancias que han variado esta estructura: a) momentos-eventos de la fase 1 que fueron aprovechados como oportunidad para avanzar en aspectos focalizados del estudio; b) momentos o circunstancias de la fase dos que fueron tomadas para generar un conocimiento holístico; c) momentos o circunstancias de cualquiera de estas dos fases que pueden entenderse como «devolución o aporte». Por tanto, lo importante no es la estructura de fases como algo sincrónico a seguir, sino desde el punto de vista de los objetivos que cada una de ellas tiene.

\subsection{Informantes}

En todos los casos los informantes han sido profesores o profesoras de los centros seleccionados, que han sido escogidos de acuerdo con los siguientes criterios: a) antigüedad; b) cargo/función: miembro del equipo directivo, tutor, jefe de departamento, miembro del equipo de orientación, o profesor sin ninguno de los cargos/funciones antes mencionadas; c) especialidad; y d) disponibilidad para observación y entrevistas.

\section{Resultados}

Los resultados son presentados de acuerdo con los 5 niveles antes explicados. Se presentan integrando los aspectos comunes a los seis casos abordados, dado que es un estudio de caso instrumental, en el que la finalidad es la comprensión del fenómeno de cambio antes explicado. Sin embargo, se ofrecerán las tonalidades específicas cuando ellas existan y sean un aporte para la comprensión mencionada.

\subsection{Currículo prescrito, nivel normativo}

Los resultados correspondientes a este nivel de acceso al objeto de estudio, se refieren a las opiniones o concepciones que los informantes tienen sobre la normativa que regula la incorporación de las competencias básicas.

Una primera tendencia a destacar de los relatos, es aquella en la que el cambio normativo es vivido con cierta indiferencia, asumiéndolo como un cambio más de los muchos que han tenido lugar en los últimos años. Incluso, para muchos de ellos, la norma que incorpora las competencias básicas no supone ninguna novedad. En esta misma línea, se aprecia otra tendencia en la que sí se detecta una novedad en la propuesta normativa de las competencias, pero se considera que no ofrece pautas ni orientaciones suficientes para plasmarla. En este sentido, los 
informantes sostienen que los Reales Decretos que prescriben las competencias no contribuyen a visualizar su concreción y a que tengan un peso real en relación a los otros componentes del currículum. El análisis de la normativa corrobora este punto de vista; en él se aprecia la coexistencia de lógicas encontradas o yuxtapuestas. Hay que aclarar también, que en uno de los centros escogidos se aprecian ciertos aspectos diferenciados con respecto al resto. Con respecto a este aspecto en concreto, consideran que la normativa es suficiente para poder actuar a partir de ella. Es necesario decir que se trata de un centro en el que ya se había optado por incorporar el enfoque de competencias antes de que saliera la norma.

Por otra parte, en el discurso de los informantes, se aprecia una actitud de protección ante las propuestas normativas por parte del profesorado, que se traduce en la ausencia de un contacto profundo con ellas. Las justificaciones que aparecen en los relatos de los entrevistados se centran en los numerosos cambios normativos de la última década, que les hacen dudar de las verdaderas intenciones relacionadas con los cambios que estas suponen. El profesorado considera que ya se le han solicitado grandes esfuerzos en reiteradas oportunidades, muchas veces sin que se diera el tiempo necesario para consolidarlos, quedando frecuentemente interrumpidos.

Otro aspecto importante que surge en primer lugar a partir del análisis de los documentos normativos, y que aparece corroborado en las entrevista, es lo que podría denominarse «lógica de repetición», según la cual, cada nueva normativa que hace alusión al tema, vuelve a repetir exactamente lo mismo que la anterior, sin ofrecer ningún grado de concreción con respecto a la que precede. Este aspecto volverá a mencionarse en los niveles de análisis siguientes.

Con respecto a la normativa, concretamente al proceso de su elaboración, en los relatos de los entrevistados se cuestiona la lógica vertical de la propuesta de cambio. Esta percepción por parte del profesorado parece estar actuando como obstáculo en la implementación o desarrollo, ya no solo del enfoque de competencias, sino también de cualquier otro tipo de propuesta. Complementariamente, esta percepción de una continua actuación vertical por parte de la Administración, aparece en los relatos representaciones que muestran una ruptura, separación o distancia significativa entre la Administración, los centros educativos y el profesorado. En este sentido, una parte importante de los entrevistados considera que a las leyes les falta un importante anclaje con la realidad, rasgo que las hace difícilmente viables para ellos.

Cerrando este nivel de análisis, es necesario mencionar algunas pequeñas diferencias entre los casos analizados. Una primera de ellas tiene que ver con las apreciaciones del profesorado según la etapa educativa de los centros. De acuerdo 
con este criterio, se puede apreciar mayor conocimiento, tanto de la normativa como de la temática de las competencias, en el profesorado de educación primaria. Por otra parte, dentro de estos centros había uno que, como ya se ha comentado, había optado por trabajar bajo el enfoque de competencias con anterioridad a la normativa; por lo tanto, su conocimiento, valoración y receptividad de las competencias era mucho mayor que en los otros casos.

\subsection{Acciones desarrolladas por la Administración para incorporar las competencias clave}

En este caso, los resultados que se exponen se desprenden de las representaciones del profesorado que aparecen en los relatos; no se han utilizado otras técnicas ni procedimientos orientados a identificarlas. Lo más llamativo que se desprende de las entrevistas es que, para la mayoría del profesorado entrevistado, la Administración no ha acompañado la normativa que prescribe la incorporación de las competencias, con otras acciones de asesoramiento, orientación, información o formación.

En el proceso de implementación de las competencias básicas aparece un actor clave, la inspección educativa, que precisamente hace de nexo entre la prescripción normativa y su concreción en los centros educativos. En la mayoría de los relatos, su presencia aparece con papel centrado en la vigilancia normativa, la cual es apreciada como la supervisión de la inclusión de las prescripciones normativas en los documentos de los centros. Este es un aspecto que el profesorado suele asociar con la burocratización de su trabajo, entendiendo por tal, la exigencia de aumentar los esfuerzos docentes en tareas relacionadas con la elaboración de diversos documentos escritos.

Por último, con respecto a las acciones de implementación que promueve la Administración, aparece un elemento que es demandando por el profesorado: la formación continua, pero que, según ellos, ha estado poco o nada presente en el proceso de implementación de las competencias básicas. Sin embargo, una vez más, se aprecian tonalidades relacionadas con características específicas de los informantes, y también de la etapa educativa. Por un lado, en cuanto a la formación inicial, aquellos que habían terminado sus estudios más recientemente, sí habían recibido algo de formación relacionada con las competencias, sobre todo el profesorado de primaria. Por otra parte, en cuanto a cursos de formación continua, al parecer, por la información aportada, estos sí existieron; el asunto es que esta formación ha sido de carácter voluntario y muchas veces permanece desconectada de lo que sucede en los centros; por lo tanto, el aprovechamiento de la misma se sitúa a un nivel individual y no institucional. 


\subsection{Acciones desarrolladas por los Centros para incorporar las competencias clave}

En este nivel de análisis se analizaron diversos procesos realizados por los centros con el fin de incorporar las competencias a su trabajo cotidiano. En la mayoría de los casos, los mismos se relacionan con la elaboración de los documentos de centro que constituyen una supuesta instancia de concreción de las prescripciones normativas. Esta es, sin duda, la práctica más extendida en este sentido. Según el análisis de estos documentos y de los relatos de los entrevistados, aparece nuevamente la lógica de la repetición mencionada en el nivel 1. De acuerdo con esta lógica, las programaciones y otros documentos de centro incluyen exactamente lo mismo, casi textual, que aquello que aparece en la ley. De esta manera, este supuesto proceso de concreción queda caracterizado por una lógica formal, no preocupada por lo que realmente va a suceder posteriormente en las aulas, sino por responder a las supuestas demandas de supervisión de la inspección. Así, el proceso de elaboración de documentos termina caracterizado por el «copiar y pegar» lo que dice la normativa, más que por procesos reflexivos relacionados con la implementación de un cambio educativo vinculado con la temática en cuestión.

Según se ha podido ver, este rasgo de la elaboración de los documentos influye en otras dinámicas que se generan y caracterizan el trabajo colaborativo del profesorado, o en su ausencia. De acuerdo con las entrevistas y las observaciones realizadas, el acento puesto en los aspectos formales de la elaboración de documentos no demanda otro tipo de acciones participativas ni colaborativas; de ahí también la ausencia significativa de debates. En este sentido, quitando el primer año de la propuesta normativa, los órganos de gobierno o de coordinación docente apenas abordaron este asunto de forma deliberativa, rasgo que se ha dado de forma casi generalizada en todos los centros, con la identificación de algunas tonalidades que se detallarán más adelante.

La información recogida permite relacionar esta lógica de repetición en la elaboración de los documentos y el gran esfuerzo dedicado a estas tareas, en la forma en la que la inspección educativa asume su propio rol. De esta manera, se ha podido constatar que la actuación de los inspectores se centraba en la supervisión, proceso que, a su vez, se centraba en el control de los documentos elaborados por los centros. Asimismo, este proceso se realizaba con un «formulario de control» en el cual se establecían aquellos aspectos que debían contener los documentos, y se marcaban a medida que los inspectores los detectaban en los mismos. Según mencionaron los entrevistados, siendo así el proceso de seguimiento de la inspección, el esfuerzo del profesorado se concentraba en la elaboración de los documentos, y el reflejo 
fiel de lo establecido por la normativa, mediante su misma copia si fuera necesario, era lo que más garantizaba superar con éxito la supervisión.

Antes de finalizar este nivel de análisis, es necesario mencionar que, más allá de estas tendencias más o menos generalizadas, se aprecian ciertas tonalidades a nivel de centros. En dos de los casos, el centro ha aprovechado el cambio normativo para debatir sobre la enseñanza e intentar implementar nuevos proyectos. En ambos, esto se ha dado al inicio de la propuesta de cambio, aunque posteriormente no se ha continuado en esta línea. Por otra parte, dos de los centros han aprovechado para desarrollar proyectos relacionados con competencias específicas, que dieron lugar a líneas de trabajo que continúan en la actualidad. Finalmente, además de estas pequeñas tonalidades de centros, también se perciben estas entre los profesores; en este sentido, en los seis centros se han identificado profesores o profesoras especialmente activos en el desarrollo de proyectos que, aunque en la mayoría de los casos no era apreciado así por el profesorado, tenían gran relación con el enfoque pedagógico de competencias.

\subsection{Currículo en acción}

Este nivel de análisis corresponde a la concreción del enfoque de competencias en los centros y en las aulas, y es el que nos permite ver en qué medida una pretensión normativa logra tener efectos en las prácticas educativas. En cinco de los seis casos estudiados, de acuerdo con la información que se desprende de las observaciones y las entrevistas, se aprecia que el enfoque de competencias no ha logrado incorporarse de forma sistemática, continuada y explícita a las prácticas docentes. En el mismo profesorado de estos cinco centros, y también en el otro caso en el que sí se aprecia su incorporación, la opinión más abundante que se encuentra en los relatos de los informantes es que el enfoque de competencias no ha producido cambios en el trabajo del aula. Sin embargo, de acuerdo con lo observado, es preciso reconocer que existen algunas excepciones. Ciertas formas del trabajo docente sí podrían tener una relación clara con el enfoque de competencias aunque, en estos casos, el profesorado no lo aprecia de esta manera, generalmente porque lo venía haciendo desde antes del cambio normativo y no establece el posible nexo conceptual con el enfoque de competencias. Este es el caso del centro que ya había incorporado el enfoque de competencias tiempo de antes de que saliera la normativa específica que lo prescribía.

\subsection{Currículo evaluado}

Este nivel, junto con el anterior, son los que ofrecen menos evidencias de la incorporación del enfoque de competencias a las prácticas de aula. Existe coincidencia en los informantes de los seis centros en que la evaluación es el 
componente que se visualiza con menos claridad en la normativa. En este sentido, con respecto a la evaluación de las competencias, el profesorado se encuentra entre los viejos conceptos que ya tiene incorporados y una nueva terminología sobre la que no tiene fundamentación conceptual. De esta manera, se aprecia un importante nivel de confusión, el cual, de acuerdo con los mismos informantes, tiene su origen en la misma propuesta normativa, que ha yuxtapuesto la estructura curricular anterior con las competencias como nuevo componente, sin mayores aclaraciones. Dentro de estas confusiones aparece con fuerza una especie de dualismo entre competencias y contenidos, que se traslada a la evaluación a modo de opción: o se evalúa por contenidos o se evalúa por competencias. Desde este punto de vista, si de opción se trata, la evaluación sigue estando enfocada en los contenidos curriculares; se aclara que, cuando se dice esto, principalmente se hace referencia a una evaluación centrada en conceptos, en su repetición o explicación.

\section{Discusión y conclusiones}

Los resultados obtenidos nos permiten apreciar los procesos de cambio educativo desde una perspectiva amplia, teniendo en cuenta que se han contemplado cinco niveles analíticos diferentes. Esta perspectiva procesual permite poner en relación las pretensiones normativas, como objetivación de un proyecto político, con los cambios que efectivamente se producen, de acuerdo con dicho proyecto. De la misma manera, permite contemplar los cambios como procesos en los que intervienen diversos actores. En esta línea, la primera conclusión, en coincidencia con investigaciones previas (GIMENO, 2006; MURILLO; KRICHESKY, 2012; PUELLES, 2006; SARASON, 2003), es que cualquier intento de cambiar las prácticas educativas que solo tenga en cuenta las prescripciones normativas, desconociendo las características que deben tener las políticas orientadas a cambios educativos, las diferentes acciones intermedias y el papel que siempre, se quiera o no, jugarán los actores implicados, está encaminado al fracaso o, cuando menos, a no tener los efectos esperados.

De acuerdo con el modelo de análisis estipulado en esta investigación, un primer aspecto clave que aparece con cierta relevancia, se relaciona con la claridad que debe tener la norma que introduce la propuesta de cambio. En el caso estudiado, como se ha explicado, se evidencia una clara yuxtaposición de lógicas o racionalidades pedagógicas (GOODSON, 1998; MOYA; LUENGO, 2011; PERRENOUD, 2012), que no facilita su comprensión por parte del profesorado y, por tanto, no es una ayuda para la implementación de la nueva propuesta de competencias.

Otro aspecto a destacar con respecto a la norma, se relaciona con una sensación generalizada de escepticismo e incredulidad con respecto a ella y a las propuestas 
que esta pueda contener, tal como se ha evidenciado en investigaciones anteriores (ÁLVAREZ, 2008; BOLIVAR, 2008; PUELLES, 2006). Según las evidencias obtenidas, el profesorado ha desarrollado una actitud de reserva, que podría denominarse también de protección, provocada por los permanentes cambios, por la falta de continuidad de las leyes y por la falta de consenso duraderos que esta situación refleja. En este sentido, la falta de pactos duraderos, construidos junto a los profesionales de la educación y otros actores sociales, hace dudar sobre la permanencia y duración de las propuestas.

Por otra parte, con respecto a las fases que es preciso tener en cuenta en los procesos de cambio o reforma educativa (ADELMAN; WALKING-EAGLE, 2003; FULLAN, 2002; HARGREAVES, 1996, 2003), la información recogida a lo largo de la investigación pone de manifiesto que, más allá de la normativa, no se han considerado de forma sistemática otro tipo de acciones. La incorporación de las competencias a la norma, no parece haber sido acompañada de acciones previas con el profesorado; tampoco se estipuló un plan estratégico, planificado, tal como se recomienda a partir de las investigaciones de Fullan (2002), que permitiese prever un proceso racional de transformación progresiva. En cambio, parece haberse depositado una creencia exagerada en la prescripción del cambio, en la normativa, como elemento principal, y casi único, promotor del mismo.

Un actor o práctica que sí ha ocupado un lugar clave en este proceso de implementación de las competencias, ha sido la inspección educativa. Su lugar en el desarrollo de las políticas parece ser relevante, aunque el tipo de impacto depende de las características que la misma adquiera. En este caso, la información obtenida muestra que los inspectores asumieron un papel centrado principalmente en la supervisión y vigilancia, esencialmente de los documentos de centro. En cambio, no se han detectado acciones de asesoramiento y orientación, tal como recomienda la investigación sobre esta temática (CANTÓN MAYO; VÁSQUEZ FERNÁNDEZ, 2010; DOMINGO SEGOVIA, 2010).

Paradójicamente, estas acciones de vigilancia y supervisión centradas casi exclusivamente en los documentos de centro, de acuerdo con la información que se ha obtenido, puede ser interpretada como un obstáculo en el desarrollo de las propuestas de cambio. Esta insistencia en la incorporación de la normativa referida a las competencias básicas a los documentos de los centros, como si ello fuese garante de su desarrollo en las aulas, lleva a que estos centren gran parte de sus esfuerzos en esta tarea, y casi exclusivamente a ella, en lo que al cambio relacionado con las competencias básicas se refiere. A esto se suma que la elaboración de documentos suele ser realizada de forma unipersonal, generalmente 
por jefes de departamento en secundaria, o coordinadores de ciclo en primaria, y no una tarea colegiada que pudiera enriquecer el trabajo cotidiano.

Siguiendo el modelo de análisis propuesto, el proceso de cambio promovido para la incorporación de las competencias básicas, no parece haber pasado las instancias prescriptivas-normativas. Después de un curso académico de presencia sistemática en los seis centros escogidos, solo en uno, y por una decisión propia previa a la norma, se ha visto la presencia de las competencias tal como se estipula en la normativa; así, el trabajo del aula sigue siendo más o menos como antes de la misma.

De esta manera, de esta investigación surgen algunos interrogantes que pueden orientar las acciones y contribuir a buscar una lógica de acción más apropiada desde el punto de vista de la promoción de cambios educativos, tal como se viene constatando en la literatura especializada en políticas, reformas y cambio educativo (FULLAN, 2002; BUENFIL, 2006; SARASON, 2003; VIÑAO, 2006). En primer lugar, es preciso contemplar los procesos de cambio de forma sistémica, es decir, con relación a todos los componentes y prácticas que lo atraviesan (GIMENO, 1988). En segundo lugar, es preciso tener en cuenta las diversas fases y momentos que son imprescindibles para que los cambios tengan éxito, tal como han puesto de manifiesto las investigaciones previas. En este mismo sentido, es necesario contemplar el factor tiempo en varias dimensiones contextuales e institucionales. En tercer, lugar hay que destacar la necesidad de establecer mecanismos de diseño de la normativa basados en evidencias y procesos participativos. Finalmente, la implementación de políticas públicas debe tener en cuenta que, en cualquiera de los casos, las prescripciones normativas se insertan en una red de significados previos a nivel institucional y profesional, los cuales harán de filtros para la reinterpretación, la concreción, la obstaculización o la paralización de las mismas. 


\section{Política e mudança em educação: o caso das competências básicas em Espanha}

\section{Resumo}

Este artigo apresenta os resultados de uma investigação cujo objetivo foi analisar a forma como as políticas públicas produzem ou não mudanças nos âmbitos da realidade social a que se destinam. Aborda, especificamente, a mudança normativa que regulamentou a implementação da abordagem pedagógica das competências em Espanha. Assume-se uma concepção processual das políticas, estudadas aqui a partir da perspectiva de diversos atores do nivel micro. Aplicou-se uma metodologia qualitativa, realizando um estudo de casos múltiplos em escolas de educação básica e secundária de três comunidades autônomas diferentes. Os resultados mostram uma diferença considerável entre as pretensões normativas e o que realmente sucedeu dentro das salas de aula das escolas. As conclusões sugerem a necessidade de uma reflexão sobre a forma como se concebem e desenvolvem as politicas públicas.

Palavras-chave: Política educativa. Mudança educativa. Competências básicas. Reformas educativas. Estudo de casos.

\section{Policy and change in education: the ase of basic competences in Spain}

\section{Abstract}

This paper shows the results of a research study whose objective was to analyze the way in which public policies produce, or not, changes in the social environment they are aimed at. The normative change that has regulated the implementation of the competences pedagogical approach in Spain is specifically addressed. A procedural vision is adopted and the policies are analyzed from the views of several actors involved at the micro level. A qualitative method was used, and multiples case studies were carried out in primary and secondary education schools in three different autonomous communities. The results show a considerable gap between the normative intentions and what really has taken place within the classrooms of the educational schools. Conclusions suggest the need for reflecting on the way in which public policies are conceived and developed.

Keywords: Education policy. Education change. Basic competences. Education reforms. Case study. 


\section{Referências}

ADELMAN, N.; WALKING-EAGLE, K. Los docentes, el tiempo y la reforma escolar. In: HARGREAVES, A. (Comp.). Replantear el cambio educativo: un enfoque renovador. Buenos Aires: Amorrortu, 2003. p. 138-62.

ÁLVAREZ, J. M. Evaluar el aprendizaje en una enseñanza centrada en competencias. In: GIMENO, J. (Comp.). Educar por competencias, ¿qué hay de nuevo? Madrid: Morata, 2008. p. 206-33.

BOLIVAR, A. Ciudadanía y competencias básicas. Sevilla: Fundación ECOEM, 2008.

BUENFIL, R. Lógicas y sentidos inscritos en la subjetividad: políticas educativas y pistas para su transformación. In: VITAR, A. (Coord.). Políticas de educación: razones de una pasión. Buenos Aires: Niño y Dávila, 2006. p. 53-88.

CANTÓN MAYO, I.; VÁSQUEZ FERNÁNDEZ, J. L. Los procesos en gestión de calidad: un ejemplo en un centro educativo. Revista Iberoamericana sobre Calidad, Eficacia y Cambio en Educación, v. 8, n. 5, p. 59-68, 2010.

CASTELLS, M. La era de la información: economia, sociedad y cultura. Madrid: Alianza Editorial, 1997. Vol. 1: La sociedad red.

DALE, R. The state and education policy. Milton Keynes: Open University Press, 1989.

DÍEZ HERNANDO, C. La competencia social y ciudadana en la LOE. Avances en Supervisión Educativa, n. 9, 2008. Disponible en: $<\mathrm{http}$ :// www.oei.es/noticias/spip.php?article3847\&debut_5ultimasOEI=5>. Acceso en: 22 enero 2015.

DOMINGO SEGOVIA, J. Comprender y redireccionar las prácticas de asesoramiento. Revista Iberoamericana de Educación, n. 54, p. 65-83, 2010.

DONOSO, S.; DONOSO, G. Políticas de gestión de la educación pública escolar en Chile (1990-2010): una evaluación inicial. Revista Ensaio: Avaliação e Políticas Públicas em Educação, v. 17, n. 64, p. 421-48, 2009. doi:10.1590/S0104-40362009000300003

DUBET, F. El declive de la institución: profesiones, sujetos e individuos ante la reforma del Estado. Barcelona: Gedisa, 2006. 
ESPAÑA. Ministerio de Educación. Ley Orgánica 2/10006, de 3 de maio. Boletín Oficial de Estado, n, 106, 4 mayo 2006.

FULLAN, M. Los nuevos significados del cambio en la educación. Octaedro: España, 2002.

GIMENO, J. El curriculum: una reflexión sobre la práctica. Madrid: Morata, 1988.

. La educación que aún es posible. Madrid: Morata, 2005.

. De las reformas como política a las políticas de reforma. In:

GIMENO, J. (Comp.). La reforma necesaria: entre la política educativa y la práctica escolar. Madrid: Morata, 2006. p. 23-42.

GOODSON, I. Historia del curriculum: la construcción social de las disciplinas escolares. Barcelona: Pomares-Corredor, 1998.

- Teorías del cambio educativo y los contextos históricos. Revista Mexicana de Investigación Educativa, v. 15, n. 47, p. 1139-45, oct.-dic. 2010.

HARGREAVES, A. Profesorado, cultura y postmodernidad: cambian los tiempos, cambia el profesorado. Madrid: Morata, 1996.

. Replantear el cambio educativo: ampliar y profundizar la búsqueda del éxito. In: HARGREAVES, A. (Comp.). Replantear el cambio educativo: un enfoque renovador. Buenos Aires: Amorrortu, 2003. p. 23-55.

MONARCA, H. Uniendo redes: apoyo comunitario y social. In: ECHEITA, G. et al. Cómo fomentar las redes naturales de apoyo en el marco de una escuela inclusiva: propuestas prácticas. Sevilla: Eduforma, 2013. p. 131-63.

MONARCA, H.; SIMÓN, C. Orientación educativa y desarrollo de prácticas participativas con las familias. Revista Nacional e Internacional de Educación Inclusiva, v. 6, n. 3, p. 120-33, 2013.

MORIN, E. Introducción al pensamiento complejo. Barcelona: Gedisa, 2003.

MOYA, J.; LUENGO, F. (Coords.). Teoría y práctica de las competencias básicas. Madrid: Grao, 2011.

MURILLO, F. J.; KRICHESKY, G. J. El proceso del cambio escolar: una guía para impulsar y sostener la mejora de las escuelas. Revista Iberoamericana sobre Calidad, Eficacia y Cambio en Educación, v. 10, n. 1, p. 26-43, 2012. 
NICASTRO, S. La cotidianeidad de lo escolar como expresión política. In: FRIGERIO, G.; DIKER, G. (Comps.). Educar: ese acto político. Paraná (Argentina): La Hendija, 2010. p. 211-24.

PARRILlA, A. Grupos de apoyo entre docentes. Cuadernos de Pedagogía, $\mathrm{n}$. 331, p. 66-69, 2004.

PÉREZ GÓMEZ, A. La cultura escolar en la sociedad neoliberal. Madrid: Morara, 1998.

PERRENOUD, P. H. Cuando la escuela pretende preparar para la vida ¿Desarrollar competencias o enseñar otros saberes? Barcelona: Graó, 2012.

PUELLES, M. Los hitos reformistas: la viabilidad de las reformas y la perversión de las leyes. In: GIMENO, J. (Comp.). La reforma necesaria: entre la política educativa y la práctica escolar. Madrid: Morata, 2006. p. 61-80.

PUJALÀS, P. 9 Ideas clave sobre el aprendizaje cooperativo. Barcelona: Graó, 2009.

RAMÍREZ, M. C. Clemencia: la antropología de la política pública. Antípoda, n. 10, p. 13-17, 2010.

SARASON, S. El predecible fracaso de la reforma educativa. Barcelona: Octaedro, 2003.

SCHÜTZ, A. El problema de la realidad social. Buenos Aires: Amorrortu, 2003.

SHORE, C. La antropología y el estudio de la política pública: reflexiones sobre la «formulación» de las políticas. Antípoda, n. 10, p. 21-49, 2010.

STAKE, R. Evaluación comprensiva y evaluación basada en estándares. Barcelona: Graó, 2006.

VELASCO, H.; DÍAZ DE RADA, A. La lógica de la investigación etnográfica: un modelo para etnógrafos de la escuela. Valladolid: Trotta, 1999.

VIÑAO, A. Sistemas educativos, culturas escolares y reformas. Madrid: Morata, 2002.

.El éxito o fracaso de las reformas educativas: condicionantes, limitaciones, posibilidades. In: GIMENO, J. (Comp.). La reforma necesaria: entre la política educativa y la práctica escolar. Madrid: Morata, 2006. p. 43-60. 
VITAR, A. Las políticas y lo(s) político(s). In: VITAR, A. (Coord.). Politicas de educación: razones de una pasión. Buenos Aires: Niño y Dávila, 2006. p. 25-49.

\section{Informações dos autores}

Héctor Monarca: Doctor en educación por la Universidad Complutense de Madrid. Profesor del Departamento de Didáctica y Teoría de la Educación de la Universidad Autónoma de Madrid. Contato: hector.monarca@uam.es

Cecilia Simón: Doctora en psicología por la Universidad Autónoma de Madrid. Profesora en la misma universidad en el Departamento Interfacultativo de Psicología Evolutiva y de la Educación. Contato: cecilia.simon@uam.es

Soledad Rappoport: Magíster en calidad y mejora de la educación y doctoranda en educación en la Universidad Autónoma de Madrid. Profesora en la misma universidad en el Departamento de Didáctica y Teoría de la Educación. Contato: soledad.rappoport@uam.es

Gerardo Echeita: Doctor en psicología por la Universidad Autónoma de Madrid. Profesor en la misma universidad en el Departamento Interfacultativo de Psicología Evolutiva y de la Educación. Contato: es/gerardo.echeita@uam.es 